\title{
Indicadores antropométricos e hemodinâmicos associados à hipertensão arterial de
}

\section{sedentários}

\author{
Anthropometric and hemodynamic indicators associated with arterial hypertension in sedentary \\ people
}

Indicadores antropométricos y hemodinámicos asociados a la hipertensión arterial en personas

sedentarias

Recebido: 28/05/2021 | Revisado: 02/06/2021 | Aceito: 05/06/2021 | Publicado: 20/06/2021

\author{
Reginaldo Luiz do Nascimento \\ ORCID: https://orcid.org/0000-0001-6467-0335 \\ Universidade Tiradentes, Brasil \\ E-mail: nascimentoreginaldoluiz@gmail.com \\ Ferdinando Oliveira Carvalho \\ ORCID: https://orcid.org/0000-0003-0306-5910 \\ Universidade Federal do Vale do São Francisco, Brasil \\ E-mail: ferdinando.carvalho@univasf.edu.br \\ Flavio de Souza Araujo \\ ORCID: https://orcid.org/0000-0001-9805-1196 \\ Universidade Federal do Vale do São Francisco, Brasil \\ E-mail: flavio.araujo@univasf.edu.br \\ Denise de Melo-Marins \\ ORCID: https://orcid.org/0000-0002-5216-6446 \\ Faculdade Uninassau, Brasil \\ E-mail: dnz_marins@hotmail.com \\ Marcos Vinicius Oliveira Carneiro \\ ORCID: https://orcid.org/0000-0002-5576-3449 \\ Faculdade Fai Irecê, Brasil \\ E-mail: maviolli@hotmail.com \\ Layane Costa Saraiva \\ ORCID: https://orcid.org/0000-0001-5151-7294 \\ Instituto Federal de Educação, Ciência e Tecnologia do Piauí, Brasil \\ E-mail: layanesaraiva@hotmail.com \\ Sérgio Rodrigues Moreira \\ ORCID: https://orcid.org/0000-0002-3068-5093 \\ Universidade Federal do Vale do São Francisco, Brasil \\ E-mail: sergio.moreira@univasf.edu.br \\ José Roberto Andrade do Nascimento Junior \\ ORCID: https://orcid.org/0000-0003-3836-6967 \\ Universidade Federal do Vale do São Francisco, Brasil \\ E-mail: joseroberto.nascimentojunior@univasf.edu.br
}

\begin{abstract}
Resumo
O estudo investigou a associação de variáveis antropométricas e hemodinâmicas à presença de hipertensão arterial em indivíduos sedentários. Participaram do estudo 3.168 voluntários de ambos os sexos (1.189 homens e 1.979 mulheres) iniciantes de uma academia de ginástica. Foram aferidas massa corporal, estatura, circunferência da cintura (CC) e do quadril, dobras cutâneas, pressão arterial sistólica e diastólica e frequência cardíaca (FC), e calculada a relação cintura-quadril (RCQ) e cintura-estatura, pressão arterial média e índice de massa corporal (IMC). Para verificar a associação entre as variáveis e a hipertensão arterial utilizou-se a regressão logística binária (bruta e ajustada). Na análise ajustada para a hipertensão sistólica, foi observada uma associação com o peso, estatura, massa gorda, faixa etária, RCQ e hipertensão diastólica. Os indivíduos a partir dos 40 anos tiveram 2,5 vezes mais chances de ter hipertensão sistólica em relação aos de 18 a 29 anos, e sujeitos com maior massa corporal e hipertensão diastólica apresentaram 1,1 e 14,9 mais chances de desenvolver hipertensão sistólica. Enquanto na análise ajustada para hipertensão diastólica observou-se associações com a idade, peso, FC, IMC e hipertensão sistólica. E os indivíduos entre 30 a 39 anos com hipertensão sistólica tiveram 1,6 e 14,2 mais chances de ter hipertensão diastólica. Conclui-se que as variáveis antropométricas e hemodinâmicas estão associadas à presença de hipertensão sistólica ou diastólica isolada em sedentários, e que indivíduos mais velhos podem ter mais chances de apresentar hipertensão em relação aos mais jovens.
\end{abstract}


Palavras-chave: Antropometria; Fatores de risco; Pressão arterial; Comportamento sedentário; Academia de ginástica.

\begin{abstract}
The study investigated the association of anthropometric and hemodynamic variables with the presence of arterial hypertension in sedentary individuals. A total of 3,168 volunteers of both sexes (1,189 men and 1,979 women) who started a gym participated in the study. Body mass, height, waist circumference (WC) and hips, skinfolds, systolic and diastolic blood pressure and heart rate (HR) were measured, and the waist-hip ratio (WHR) and waist-height ratio were calculated, mean arterial pressure and body mass index (BMI). To verify the association between variables and arterial hypertension, binary logistic regression (crude and adjusted) was used. In the adjusted analysis for systolic hypertension, an association was observed with weight, height, fat mass, age group, WHR and diastolic hypertension. Individuals over 40 were 2.5 times more likely to have systolic hypertension than those aged 18 to 29 , and subjects with greater body mass and diastolic hypertension were 1.1 and 14.9 more likely to develop systolic hypertension. While in the adjusted analysis for diastolic hypertension, associations were observed with age, weight, HR, BMI and systolic hypertension. And individuals aged 30 to 39 years with systolic hypertension were 1.6 and 14.2 more likely to have diastolic hypertension. It is concluded that anthropometric and hemodynamic variables are associated with the presence of isolated systolic or diastolic hypertension in sedentary people, and that older individuals may be more likely to have hypertension compared to younger ones.
\end{abstract}

Keywords: Anthropometry; Risk factors; Arterial pressure; Sedentary behavior; Fitness centers.

\title{
Resumen
}

El estudio investigó la asociación de variables antropométricas y hemodinámicas con la presencia de hipertensión arterial en individuos sedentarios. En el estudio participaron un total de 3.168 voluntarios de ambos sexos (1.189 hombres y 1.979 mujeres) que iniciaron un gimnasio. Se midieron la masa corporal, la altura, la circunferencia de la cintura (CC) y las caderas, los pliegues cutáneos, la presión arterial sistólica y diastólica y la frecuencia cardíaca (FC), y se calculó la relación cintura-cadera (RCC) y cintura-altura, y la presión arterial media y índice de masa corporal (IMC). Para verificar la asociación entre variables e hipertensión arterial se utilizó regresión logística binaria (cruda y ajustada). En el análisis ajustado para la hipertensión sistólica, se observó una asociación con el peso, la talla, la masa grasa, el grupo de edad, la RCC y la hipertensión diastólica. Los individuos mayores de 40 años tenían 2,5 veces más probabilidades de tener hipertensión sistólica que los de 18 a 29 años, y los sujetos con mayor masa corporal e hipertensión diastólica tenían 1,1 y 14,9 más probabilidades de desarrollar hipertensión sistólica. Mientras que en el análisis ajustado para hipertensión diastólica, se observaron asociaciones con edad, peso, FC, IMC e hipertensión sistólica. Y las personas de 30 a 39 años con hipertensión sistólica tenían 1,6 y 14,2 más probabilidades de tener hipertensión diastólica. Se concluye que las variables antropométricas y hemodinámicas están asociadas con la presencia de hipertensión arterial sistólica o diastólica aislada en personas sedentarias, y que los individuos mayores pueden tener mayor probabilidad de tener hipertensión en relación a los más jóvenes.

Palabras clave: Antropometría; Factores de riesgo; Presión arterial; Comportamiento sedentario; Gimnasio.

\section{Introdução}

A hipertensão arterial (HA) é multifatorial e considerada um dos principais fatores de risco para doenças cardiovasculares (DCV) e renais, possuindo impacto significativo nos custos médicos e socioeconômicos no Brasil e no mundo (Barroso et al., 2021; Malta et al., 2017). Esta condição crônica é prevalente em adultos brasileiros (24,5\%) (Brasil, 2020) e comumente associada ao histórico familiar, idade, sexo, entre outros fatores que podem ser modificáveis como dieta, sedentarismo, sobrepeso/obesidade geral e obesidade abdominal (Barroso et al., 2021; Oliveira et al., 2017).

As taxas de obesidade quase triplicaram desde 1975 em países desenvolvidos e em desenvolvimento, podendo aumentar as morbimortalidades por DCV de formar direta e indiretamente (WHO, 2020; Koliaki et al., 2019). Em paralelo, tem-se estudado tanto os fatores determinantes da obesidade e HA (Santos et al., 2020) para reduzir as morbidades cardiovasculares (Santos Dias et al., 2021), quanto indicadores antropométricos para estimar o risco coronariano de diferentes populações (Loureiro et al., 2020; Nascimento et al., 2021).

A utilização de indicadores antropométricos de obesidade tem mostrado fácil aplicabilidade, baixo custo, bons resultados na identificação de riscos cardiovasculares (Ferriani et al., 2017; Barroso et al., 2020) e ampla utilização na prática clínica e em pesquisas populacionais. E alguns estudos têm apontado uma relação entre variáveis antropométricas e aumentos significativos na pressão arterial (PA) de repouso entre homens e mulheres independente da idade (Nascimento et al., 2017; 
Pohl et al., 2018).

Muitas investigações fizeram associações de medidas antropométricas com risco cardiovascular, mas conforme nosso conhecimento, ainda não foi utilizado uma amostra numerosa de sedentários frequentadores iniciantes de uma academia de ginástica. Uma vez que a vigilância efetiva das morbidades cardiovasculares é assimilada mediante a avaliação e identificação precoce dos riscos nos indivíduos, permitindo o acompanhamento e intervenção adequada. Assim, o presente estudo teve como objetivo investigar a associação de variáveis antropométricas e hemodinâmicas à presença de HA em indivíduos sedentários.

\section{Metodologia}

Trata-se de um estudo quantitativo com delineamento transversal, integrado ao projeto de extensão "Academia Universitária" sob parecer $n^{\circ}$ 5931.0.000.441-10 do Comitê de Ética em Pesquisa da Universidade Federal do Vale do São Francisco (UNIVASF), Petrolina, Pernambuco, Brasil. A pesquisa atendeu todas as normas éticas dispostas na Lei $\mathrm{n}^{\circ} 466 / 12$ do Conselho Nacional de Saúde e foi realizada no período de janeiro de 2010 a dezembro de 2016.

A amostra incluiu 3.168 indivíduos de ambos os sexos, dos quais 1.189 homens e 1.979 mulheres com idade entre 18 a 71 anos, autodeclarados sedentários (com no mínimo seis meses de inatividade física antes da avaliação física inicial) e frequentadores iniciantes de uma academia de ginástica na cidade de Petrolina - PE. A seleção da amostra foi realizada de forma aleatória, por meio de convite no ato da inscrição e/ou matrícula na academia de ginástica, sendo incluídas pessoas consideradas sedentárias e excluídas as que não se dispuseram a realizar a avaliação física.

Os participantes foram informados das características gerais do estudo, assim como orientados a não consumirem bebidas alcoólicas ou à base de cafeína em um período de 24 horas antes da primeira avaliação física, e recomendou-se a manutenção das atividades habituais no período da pesquisa.

Os dados foram coletados na avaliação física inicial dos participantes, sendo mensuradas medidas antropométricas e hemodinâmicas: massa corporal $(\mathrm{kg})$, estatura $(\mathrm{m})$, circunferência da cintura $(\mathrm{cm})$ e do quadril $(\mathrm{cm})$, dobras cutâneas, pressão arterial sistólica (PAS) e diastólica (PAD) e frequência cardíaca (FC), e posteriormente calculada o índice de massa corporal (IMC), relação cintura-quadril (RCQ) e cintura-estatura (RCE) e pressão arterial média (PAM). Todos os procedimentos que envolveram as coletas dos dados foram executados por um único avaliador treinado e experiente, com o nível de confiabilidade de $90 \%$ (Silva et al., 2011).

A variável massa corporal foi avaliada por meio de uma balança de plataforma digital (Tec-Silver, Techline, Brasil) com resolução de $0,1 \mathrm{~kg}$, e a estatura verificada por um estadiômetro (ES2030, Sanny, Brasil) com resolução de 0,1 cm, de acordo com procedimentos previamente descritos (Gordon et al., 1988). O IMC foi calculado por meio da fórmula: [massa corporal $(\mathrm{kg}) /$ estatura $\left.(\mathrm{m})^{2}\right]$.

A circunferência da cintura (CC) foi medida no ponto médio entre a última costela e a crista ilíaca e a circunferência do quadril (CQ) no ponto de maior circunferência da região glútea, por meio de uma fita métrica metálica inextensível (escala de 0,1 cm, Cescorf, Brasil) seguindo técnicas padronizadas (Callaway, 1988). A RCQ foi calculada pela razão da CC pela CQ e a RCE por meio da razão da CC pela estatura, sendo utilizadas para estimar o risco coronariano a CC e a RCE conforme pontos de corte (Pitanga, 2011).

Para aferição das dobras cutâneas nos homens (abdominal, peitoral e coxa medial) e nas mulheres (coxa medial, triciptal e suprailíaca) foi utilizado um adipômetro (ME01263A, Cescorf, Brasil) conforme protocolo de três dobras (Slaughter et al., 1984).

A PA e FC foram mensuradas com a utilização de um aparelho oscilométrico digital (BP 3AC1-1, Microlife, USA) devidamente calibrado e validado (Topouchian et al., 2005). As medidas de PAS, PAD e FC foram padronizadas no braço esquerdo, com o avaliado sentado em um local calmo (sala de avaliação física) e após um período de 10 minutos de repouso 
(Malachias et al., 2016). A classificação da amostra para HA sistólica isolada (PAS $\geq 140 \mathrm{mmHg}$ e PAD $<90 \mathrm{mmHg}$ ) e HA diastólica isolada (PAS $<140 \mathrm{mmHg}$ e PAD $\geq 90 \mathrm{mmHg}$ ) seguiu as diretrizes brasileiras de hipertensão arterial - 2020 (Barroso et al., 2021).

A análise foi realizada mediante uma abordagem de estatística descritiva e inferencial. As variáveis numéricas foram expressas por meio da média e desvio-padrão. Na estatística inferencial, a Regressão Logística binária (bruta e ajustada) foi usada para examinar as associações das variáveis antropométricas e hemodinâmicas dos indivíduos sedentários com a presença de HA (variável dependente). O ajuste do modelo foi verificado por meio do teste de Hosmer-Lemeshow. Todas as variáveis foram para a análise ajustada, independentemente do p-valor na análise bruta. Na análise ajustada, foi adotada abordagem hierárquica, seguindo o procedimento backward de introdução das variáveis nos modelos. Adotou-se como critério para permanência do fator nas análises de regressão ajustada um p-valor inferior a 0,20. No fim, chegou-se a um modelo final de regressão com apenas aquelas variáveis de maior significância estatística. As análises dos dados foram realizadas no Software SPSS 22.0, e considerado um nível de significância de p < 0,05 e intervalo de confiança (IC) de 95\%, com cálculo dos odds ratios ajustados.

\section{Resultados}

A Tabela 1 apresenta as características antropométricas e hemodinâmicas da amostra, dos quais metade dos avaliados estava acima do peso (36,6\% com sobrepeso e 13,5\% com obesidade), uma parcela apresentou maior risco cardiovascular conforme a CC (21,6\% risco moderado e 8,5\% risco aumentado) e RCE (21,7\% risco moderado e 11,6\% alto risco). Além disso, alguns apresentaram risco aumentado de acordo com a RCQ (9,4\%) e HA sistólica isolada (10,2\%) e diastólica isolada $(13,2 \%)$ segundo valores de PAS e PAD.

Tabela 1 - Perfil antropométrico e hemodinâmico da amostra (Petrolina - PE, n = 3.168).

\begin{tabular}{|c|c|}
\hline Variáveis & \\
\hline Massa corporal $(\mathrm{x} \pm \mathrm{dp})$ & $69,22 \pm 14,05$ \\
\hline Estatura (x \pm dp) & $1,65 \pm 0,09$ \\
\hline IMC (x \pm dp $)$ & $25,28 \pm 4,15$ \\
\hline $\mathrm{CQ}(\mathrm{x} \pm \mathrm{dp})$ & $99,76 \pm 16,40$ \\
\hline $\mathrm{RCQ}(\mathrm{x} \pm \mathrm{dp})$ & $0,82 \pm 1,32$ \\
\hline $\mathrm{CC}(\mathrm{x} \pm \mathrm{dp})$ & $79,80 \pm 10,77$ \\
\hline $\operatorname{RCE}(x \pm d p)$ & $48,31 \pm 6,06$ \\
\hline$\% \mathrm{G}(\mathrm{x} \pm \mathrm{dp})$ & $27,70 \pm 9,50$ \\
\hline Massa magra $(x \pm d p)$ & $49,81 \pm 11,34$ \\
\hline Massa gorda $(x \pm d p)$ & $19,44 \pm 8,39$ \\
\hline $\mathrm{FC}(\mathrm{x} \pm \mathrm{dp})$ & $79,38 \pm 16,88$ \\
\hline $\operatorname{PAS}(x \pm d p)$ & $122,46 \pm 13,84$ \\
\hline $\operatorname{PAD}(x \pm d p)$ & $78,43 \pm 9,69$ \\
\hline PAM (x $\pm d p)$ & $93,10 \pm 10,23$ \\
\hline \multicolumn{2}{|l|}{ IMC (f / \%) } \\
\hline Baixo peso & $83 / 2,6$ \\
\hline Peso normal & 1499 / 47,3 \\
\hline
\end{tabular}


Sobrepeso

Obesidade

$\mathrm{CC}(\mathrm{f} / \%)$

Baixo risco

Risco moderado

Risco alto

$\mathrm{RCE}(\mathrm{f} / \%)$

Baixo risco

Risco moderado

Risco alto

$\mathrm{RCQ}(\mathrm{f} / \%)$

Baixo risco

Risco aumentado

HA sistólica isolada

Normotenso

Hipertenso

HA diastólica isolada

Normotenso

Hipertenso
$1158 / 36,6$

428 / 13,5

$2217 / 69,9$

$686 / 21,6$

$270 / 8,5$

$2115 / 66,7$

$690 / 21,7$

$368 / 11,6$

$2875 / 90,6$

$298 / 9,4$

$2848 / 89,8$

$325 / 10,2$

$2753 / 86,8$

$420 / 13,2$

IMC = Índice de massa corporal; $\mathrm{CQ}=$ Circunferência do quadril; $\mathrm{RCQ}=$ Relação cintura-quadril; $\mathrm{CC}=$ Circunferência da cintura; $\mathrm{RCE}=$ Relação cintura-estatura; \%G = Percentual de gordura; FC = Frequência cardíaca; PAS = Pressão arterial sistólica; PAD = Pressão arterial diastólica; PAM = Pressão arterial média; HA = Hipertensão arterial. Fonte: Dados da pesquisa (2017).

Ao analisar a associação (bruta e ajustada) da presença de HA sistólica isolada com variáveis antropométricas e hemodinâmicas dos voluntários (Tabela 2), verificou-se na análise bruta associação significativa $(\mathrm{p}<0,05)$ da HA sistólica isolada com a idade, peso, estatura, massa gorda, sexo, faixa etária, IMC, CC, RCE, RCQ e HA diastólica isolada, e na análise ajustada, associação $(\mathrm{p}<0,05)$ apenas com o peso, estatura, masssa gorda, faixa etária, RCQ e HA diastólica isolada.

Tabela 2 - Associação entre variáveis e presença de hipertensão arterial sistólica isolada de sedentários (Petrolina - PE).

\begin{tabular}{lcccc}
\hline Variáveis & $\mathrm{n}$ & $\mathrm{HA}(\mathrm{PAS})$ & OR $_{\text {bruta }}[\mathrm{IC95 \%}]$ & OR $_{\text {ajustada }}[\mathrm{IC95 \%}]$ \\
\hline Idade $(\mathrm{x} \pm \mathrm{dp})$ & 3168 & $34,02 \pm 10,89$ & $1,04[1,03-1,06]^{*}$ & - \\
Massa corporal $(\mathrm{x} \pm \mathrm{dp})$ & 3168 & $78,75 \pm 14,65$ & $1,04[1,04-1,05]^{*}$ & $1,10[1,06-1,14]^{*}$ \\
Estatura $(\mathrm{x} \pm \mathrm{dp})$ & 3168 & $1,68 \pm 0,10$ & $67,77[19,56-234,80]^{*}$ & $0,00[0,00-0,15]^{*}$ \\
CQ $(\mathrm{x} \pm \mathrm{dp})$ & 3168 & $101,95 \pm 8,90$ & $1,00[0,99-1,01]$ & - \\
\%G (x $\pm \mathrm{dp})$ & 3168 & $26,86 \pm 9,64$ & $0,99[0,97-1,00]$ & - \\
Massa magra $(\mathrm{x} \pm \mathrm{dp})$ & 3168 & $57,53 \pm 12,43$ & $1,00[1,00-1,00]$ & - \\
Massa gorda $(\mathrm{x} \pm \mathrm{dp})$ & 3168 & $21,09 \pm 8,73$ & $1,02[1,01-1,03]^{*}$ & $0,94[0,91-0,97]^{*}$ \\
FC (x \pm dp) & 3168 & $79,70 \pm 12,82$ & $1,00[0,99-1,00]$ & - \\
Sexo (f / \%) & & & & 1,00
\end{tabular}


Feminino

Faixa etária

18 a 29 anos
30 a 39 anos

40 a 49 anos

50 anos ou mais

IMC (f / \%)

Baixo peso

Peso normal

Sobrepeso

Obesidade

CC (f / \%)

\section{Baixo risco}

Risco moderado

Risco alto

RCE (f / \%)

Baixo risco

Risco moderado

Risco alto

RCQ (f / \%)

Baixo risco
Risco aumentado

HA diastólica isolada

Normotenso
Hipertenso

1979

$116(5,9)$

1793

$136(7,6)$

878

$84(9,6)$

$74(20,0)$

$31(24,4)$

$1(1,2)$

1499

$91(6,1)$

1158

$150(13,0)$

428

$83(19,3)$

2214

$211(9,5)$

684

$72(10,5)$

270

$42(15,6)$

2111

689

368

$196(9,3)$

$78(11,3)$

$51(13,9)$

2871

$270(9,4)$

297

$55(18,5)$

$127(4,6)$

1,00

0,63 [0,37-1,07]

$0,29[0,22-0,37]^{*}$

1,00

$$
\begin{gathered}
1,29[0,97-1,71] \\
3,05[2,24-4,15]^{*} \\
3,94[2,53-6,13]^{*}
\end{gathered}
$$

0,82 [0,57-1,16]

2,51 [1,67-3,77]*

$2,52[1,43-4,43]^{*}$

1,00

$5,30[0,72-38,50]$

2,67 [0,34-20,90]

12,20 [1,68-88,31]*

$1,67[0,20-13,96]$

19,72 [2,70-143,79]*

$0,92[0,09-8,86]$

1,00

1,00

1,11 [0,84-1,47]

$1,75[1,22-2,50]^{*}$

1,00

1,00

1,24 [0,94-1,64]

1,57 [1,13-2,19]*

1,00

1,00

$2,18[1,58-3,00]^{*}$

$0,62[0,39-0,97]^{*}$

1,00

1,00

$420 \quad 198(47,1)$

$18,44[14,19-23,85]^{*}$

$14,914[11,18-19,88]^{*}$

$\mathrm{CQ}=$ Circunferência do quadril; \%G = Percentual de gordura; FC = Frequência cardíaca; $\mathrm{IMC}=$ Índice de massa corporal; CC = Circunferência da cintura; $\mathrm{RCE}=$ Relação cintura-estatura; $\mathrm{RCQ}=$ Relação cintura-quadril; HA = Hipertensão arterial. *Associação significativa $=$ p $<0,05$ : Regressão Logística Binária. OR = Odds Ratio; $\mathrm{IC}=$ Intervalo de confiança. OR ajustada por todas as variáveis, independente do valor de $p$ na análise bruta. Fonte: Dados da pesquisa (2017).

Os indivíduos a partir dos 40 anos tiveram 2,5 vezes mais chances de apresentar HA sistólica isolada em relação aos sujeitos com idade entre 18 e 29 anos, e voluntários com maior massa corporal e HA diastólica isolada apresentaram 1,1 [IC95\% = 1,06-1,14] e 14,9 [IC95\% = 11,18-19,88] vezes mais chances de ter HA sistólica isolada. Entretanto, pessoas com baixo risco para RCQ e menor quantidade de massa gorda apresentaram 38\% e 5\% mais chances de ter HA sistólica isolada.

Em relação à associação da presença de HA diastólica isolada com as variáveis (Tabela 3), foi verificada na análise bruta associação significativa $(\mathrm{p}<0,05)$ da HA diastólica isolada com a idade, peso, estatura, massa magra, massa gorda, FC, sexo, faixa etária, IMC, CC, RCE, RCQ e HA sistólica isolada, e para análise ajustada, houve associação $(\mathrm{p}<0,05)$ apenas com a idade, peso, FC, faixa etária, IMC e HA sistólica isolada. 
Tabela 3 - Associação entre variáveis e presença de hipertensão arterial diastólica isolada de sedentários (Petrolina - PE).

\begin{tabular}{|c|c|c|c|c|}
\hline Variáveis & $\mathrm{n}$ & HA (PAD) & $\mathrm{OR}_{\text {bruta }}[\mathrm{IC} 95 \%]$ & $\mathrm{OR}_{\text {ajustada }}[\mathrm{IC} 95 \%]$ \\
\hline Idade $(\mathrm{x} \pm \mathrm{dp})$ & 3168 & $34,74 \pm 9,92$ & $1,05[1,04-1,07]^{*}$ & $1,07[1,02-1,11]^{*}$ \\
\hline Massa corporal $(x \pm d p)$ & 3168 & $76,74 \pm 15,06$ & $1,04[1,03-1,04]^{*}$ & $1,01[1,00-1,02]^{*}$ \\
\hline Estatura (x \pm dp) & 3168 & $1,67 \pm 0,10$ & $6,30[2,07-19,15]^{*}$ & - \\
\hline $\mathrm{CQ}(\mathrm{x} \pm \mathrm{dp})$ & 3168 & $101,91 \pm 7,65$ & $1,00[0,99-1,02]$ & - \\
\hline$\% \mathrm{G}(\mathrm{x} \pm \mathrm{dp})$ & 3168 & $28,50 \pm 8,64$ & $1,01[0,99-1,02]$ & - \\
\hline Massa magra $(x \pm d p)$ & 3168 & $22,01 \pm 8,32$ & $1,04[1,03-1,05]^{*}$ & - \\
\hline Massa gorda $(x \pm d p)$ & 3168 & $54,60 \pm 12,18$ & $1,04[1,02-1,05]^{*}$ & - \\
\hline $\mathrm{FC}(\mathrm{x} \pm \mathrm{dp})$ & 3168 & $81,95 \pm 12,23$ & $1,01[1,00-1,01]^{*}$ & $1,01[1,00-1,03]^{*}$ \\
\hline \multicolumn{5}{|l|}{$\operatorname{Sexo}(\mathrm{f} / \%)$} \\
\hline Masculino & 1189 & $222(18,7)$ & 1,00 & 1,00 \\
\hline Feminino & 1979 & $198(10,0)$ & $0,48[0,39-0,59]^{*}$ & - \\
\hline Faixa etária & & & & - \\
\hline 18 a 29 anos & 1793 & $141(7,9)$ & 1,00 & 1,00 \\
\hline 30 a 39 anos & 878 & $155(17,7)$ & $2,51[1,96-3,20]^{*}$ & $1,67[1,03-2,71]^{*}$ \\
\hline 40 a 49 anos & 370 & $85(23,0)$ & $3,49[2,59-4,70]^{*}$ & $1,02[0,44-2,38]$ \\
\hline 50 anos ou mais & 127 & $39(30,7)$ & $5,19[3,43-7,85]^{*}$ & $0,89[0,25-3,11]$ \\
\hline \multicolumn{5}{|l|}{$\mathrm{IMC}(\mathrm{f} / \%)$} \\
\hline Baixo peso & 83 & $8(9,6)$ & 1,00 & 1,00 \\
\hline Peso normal & 1499 & $102(6,8)$ & $0,68[0,32-1,45]$ & $0,34[0,15-0,78]^{*}$ \\
\hline Sobrepeso & 1158 & $197(17,0)$ & $1,92[0,91-4,04]$ & $0,67[0,27-1,66]$ \\
\hline Obesidade & 428 & $113(26,4)$ & $3,36[1,57-7,19]^{*}$ & $0,87[0,30-2,53]$ \\
\hline \multicolumn{5}{|l|}{$\mathrm{CC}(\mathrm{f} / \%)$} \\
\hline Baixo risco & 2214 & $264(11,9)$ & 1,00 & 1,00 \\
\hline Risco moderado & 684 & $93(13,6)$ & $1,16[0,90-1,49]$ & - \\
\hline Risco alto & 270 & $63(23,3)$ & $2,24[1,64-3,06]^{*}$ & - \\
\hline \multicolumn{5}{|l|}{ RCE (f / \%) } \\
\hline Baixo risco & 2111 & $244(11,6)$ & 1,00 & 1,00 \\
\hline Risco moderado & 689 & $101(14,7)$ & $1,31[1,02-1,68]^{*}$ & - \\
\hline Risco alto & 368 & $75(20,4)$ & $1,95[1,47-2,60]^{*}$ & - \\
\hline \multicolumn{5}{|l|}{ RCQ (f / \%) } \\
\hline Baixo risco & 2871 & $342(11,9)$ & 1,00 & 1,00 \\
\hline Risco aumentado & 297 & $78(26,3)$ & $2,63[1,98-3,49]^{*}$ & - \\
\hline \multicolumn{5}{|l|}{ HA sistólica isolada } \\
\hline Normotenso & 2748 & $222(7,8)$ & 1,00 & 1,00 \\
\hline Hipertenso & 420 & $198(60,9)$ & $18,40[14,17-23,91]^{*}$ & $14,26[10,72-18,98]^{*}$ \\
\hline
\end{tabular}

$\mathrm{Q}=$ Circunferência do quadril; \%G = Percentual de gordura; FC = Frequência cardíaca; IMC = Índice de massa corporal; CC = Circunferência da cintura; $\mathrm{RCE}=$ Relação cintura-estatura; $\mathrm{RCQ}=$ Relação cintura-quadril; $\mathrm{HA}=$ Hipertensão arterial. $*$ Associação significativa $=\mathrm{p}<0,05$ : Regressão Logística Binária. OR = Odds Ratio; IC = Intervalo de confiança. OR ajustada por todas as variáveis, independente do valor de p na análise bruta. Fonte: Dados da pesquisa (2017). 
Ressalta-se que indivíduos de 30 a 39 anos e com HA sistólica isolada tiveram 1,6 [IC95\% = 1,03-2,71] e 14,2 [IC95\% = 10,72-18,98] vezes mais chances de ter HA diastólica isolada. E os voluntários mais velhos (101\%), com maior massa corporal (102\%), maior FC (107\%) e baixo peso (65\%) possuíram mais chances de ter HA diastólica isolada.

\section{Discussão}

O presente estudo investigou a associação de variáveis antropométricas e hemodinâmicas à presença de HA em indivíduos sedentários, evidenciando que metade dos 3.168 avaliados estava acima do peso (sobrepeso/obesidade), assim como uma parcela apresentou risco cardiovascular conforme a CC e RCE, risco aumentado de acordo com a RCQ e HA sistólica ou diastólica isolada para valores de PA. Além disso, pessoas mais velhas e com maior massa corporal tiveram mais chances de ter HA.

Uma das preocupações do estudo é a relação entre o acréscimo de massa corporal e de obesidade com o aumento do risco de DCV na população, sendo que metade dos investigados na presente pesquisa estava com sobrepeso/obesidade. Uma recente revisão sistemática e meta-análise (Jayedi et al., 2018) evidenciou que o risco de hipertensão aumentou continuamente com o ganho de peso e aumento das medidas antropométricas de IMC, CC, RCQ e RCE. E no estudo de Malta et al. (2017), no qual mais da metade dos pesquisados também eram sobrepesados/ obesos, a obesidade foi associada a maior chance de HA entre adultos brasileiros.

Na prática clínica, a perda de peso constitui uma das recomendações para melhorar o perfil do fator de risco de DCV em indivíduos com sobrepeso e obesidade (Arnett et al., 2019). E essa perda de peso ou de gordura, mesmo que não influencie de forma independente na PA, pode induzir a declínios paralelos na pressão sanguínea em decorrência de reduções na resistência à insulina, melhora da retenção de sódio, alterações na estrutura e função vascular, aumento da ativação do sistema nervoso simpático e alterações no peptídeo natriurético (Harsha \& Bray, 2008).

Os avaliados com mais idade e maior massa corporal também demonstraram mais chances de ter HA sistólica ou diastólica isolada em relação aos mais jovens e com menor massa corporal, confirmando com as diretrizes de hipertensão (Barroso et al., 2021; Oliveira et al., 2017). Assim, a idade avançada é um fator de risco não modificável para HA e a perda de peso ou de gordura é uma interveção não farmacológica para o tratamento de hipertensão, em que se espera redução de 1 mmHg para cada diminuição de $1 \mathrm{~kg}$ no peso principalmente em indivíduos com sobrepeso (Whelton et al., 2018). Os autores ainda ressaltam que a perda de peso pode impactar reduções na PAS de até $-5 \mathrm{mmHg}$ em hipertensos e $-3 \mathrm{mmHg}$ em normatensos.

A análise ajustada da HA sistólica e diastólica isolada com todas as variáveis mostrou associação com a massa corporal, idade, massa gorda, faixa etária, RCQ, FC e IMC, e os sedentários com obesidade geral apresentaram maior probabilidade de serem hipertensos que aqueles com nenhum fator de risco independente da idade e sexo. A avaliação da obesidade é imprescindível para diagnóstico e tratamento precoce a fim de evitar doenças e consequentes prejuízos à saúde, dado que a diretriz de hipertensão (Barroso et al., 2021) recomenda aos profissionais de saúde que realizem as simples medidas de CC e IMC para predizer morbidade e risco de morte, considerando-as como um importante "sinal vital” na prática clínica.

Um estudo global (Gakidou et al., 2017) destacou o IMC elevado como um dos principais riscos para causas de doenças apresentando aumentos significativos desde 1990, e outras pesquisas de intervenção (Saraiva et al., 2020) e transversal (Vrettos et al., 2020) comprovaram que os hipertensos eram majoritariamente obesos (IMC $=32,5 \pm 6,9 \mathrm{~kg} / \mathrm{m} 2$ ) e que o IMC e a hipertensão possuem um vínculo inquebrável em razão de hipertensos serem propensos a IMC aumentado.

Por outro lado, a CC é muito utilizada e considerada um bom preditor de risco para DCV e metabólicas, fato que pode ser relacionado ao acúmulo de tecido adiposo na região abdominal. No estudo de Nascimento et al. (2017) a CC apresentou 
maior correlação significativa com a PA de repouso de homens e mulheres. E Carvalho et al. (2013), que investigaram a agregação de fatores de risco cardiovascular e ocorrência de HA em adultos sedentários, identificaram associação forte entre alta prevalêcia HA, diagnóstico de obesidade e valores elevados de CC.

Algumas pesquisas mostraram outros indicadores antropométricos com poder em predizer risco cardiovascular, como a RCE em adultos trabalhadores (Ferriani et al., 2017), RCQ em trabalhadores rurais (Pohl et al., 2018), RCE e IMC em homens adultos (Loureiro et al., 2020), e CC e RCE em adultos sedentários (Nascimento et al., 2021). Com essas evidências é possível observarmos que indicadores antropométricos de baixo custo e fácil aplicabilidade são capazes de prognosticar o risco cardiovascular, sendo relevante para prevenção e agravos de doenças. Logo o controle da PA só pode ser alcançado se pessoas com hipertensão forem identificadas, diagnosticadas e tratadas (Carey et al., 2018).

O estudo apresenta algumas limitações: as informações sobre período, frequência e duração de atividades físicas foram autodeclaradas pelos avaliados classificando-os como sedentários, mas acredita-se que os dados informados sejam satisfatórios; não foi aplicado um questionário de frequência alimentar, considerando que a dieta rica em gordura e sal eleva os níveis de PA; e foi realizada a avaliação de dobras cutâneas para estimar a gordura corporal também de pessoas obesas, uma vez que esse método não é indicado para esse público.

Como pontos fortes, o estudo considerou uma amostra representativa de 3.168 avaliados, a avaliação de medidas antropométricas e hemodinâmicas foi realizada por um único avaliador favorecendo a padronização e minimização de erros técnicos, os participantes receberam as devidas orientações antes da primeira e única avaliação contribuindo para maior fidedignidade das medidas, e ter considerado vários indicadores antropométricos para estimar o risco de DCV.

\section{Considerações Finais}

Conclui-se que as variáveis antropométricas e hemodinâmicas associam-se à presença de HA sistólica ou diastólica isolada em sedentários, demonstrando que o sobrepeso/obesidade aumenta os riscos cardiovasculares. Ademais, pessoas mais velhas e com maior massa corporal podem apresentar mais chances de ter hipertensão.

Os resultados indicam aplicações práticas, como encorajar as pessoas a manterem seu peso ideal a partir dos esclarecimentos sobre os prejuízos do sobrepeso/obesidade para aumento do risco cardiovascular e nossa saúde, recomendar a simples utilização de uma fita métrica para indicar o risco cardiovascular por meio da CC, e contribuir para a identificação precoce dos fatores de risco, permitindo estratégias e ações de prevenção e controle das DCV. E recomenda-se para estudos futuros, a avaliação antropométrica e hemodinâmica em outros grupos de sedentários e de praticantes de exercício físico para uma análise mais abrangente e melhor compreensão dos resultados.

\section{Referências}

Arnett, D. K., Blumenthal, R. S., Albert, M. A., Buroker, A. B., Goldberger, Z. D., Hahn, E. J., ... \& Ziaeian, B. (2019). 2019 ACC/AHA guideline on the primary prevention of cardiovascular disease: a report of the American College of Cardiology/American Heart Association Task Force on Clinical Practice Guidelines. Journal of the American College of Cardiology, 74(10), e177-e232. https://doi.org/10.1016/j.jacc.2019.03.010

Barroso, M. L., Moura, A. M. W. A., \& Pinto, N. V. (2020). Correlação entre obesidade geral e abdominal em mulheres ativas diabéticas e/ou hipertensas. Research, Society and Development, 9(7), e179973679-e179973679. http://dx.doi.org/10.33448/rsd-v9i7.3679

Barroso, W. K. S., Rodrigues, C. I. S., Bortolotto, L. A., Mota-Gomes, M. A., Brandão, A. A., Feitosa, A. D. D. M., ... \& Nadruz, W. (2021). Diretrizes Brasileiras de Hipertensão Arterial-2020. Arquivos Brasileiros de Cardiologia, 116(3), 516-658. https://doi.org/10.36660/abc.20201238

Brasil. (2020). Vigilância de fatores de risco e proteção para doenças crônicas por inquérito telefônico. Secretaria de Vigilância em Saúde-Brasília: Ministério da Saúde. 137. http://bvsms.saude.gov.br/bvs/publicacoes/vigitel_brasil_2019_vigilancia_fatores_risco.pdf

Callaway, C. W. (1988). Circunferences. In T. G. Logman, A. F. Roche \& V. C. Seefeldt (Eds.), Anthropometric Standardization Reference Manual. Human Kinetics Books. 
Carey, R. M., Muntner, P., Bosworth, H. B., \& Whelton, P. K. (2018). Prevention and control of hypertension: JACC health promotion series. Journal of the American College of Cardiology, 72(11), 1278-1293. https://doi.org/10.1016/j.jacc.2018.07.008

Carvalho, F. O., Fernandes, R. A., Christofaro, D. G. D., Codogno, J. S., Monteiro, H. L., Moreira, S. R., ... \& Simões, H. G. (2013). Agregação de fatores de risco cardiovascular e ocorrência de hipertensão arterial em adultos sedentários. Revista Brasileira de Medicina do Esporte, 19(6), 419-422. https://doi.org/10.1590/S1517-86922013000600008

Ferriani, L. O., Martins, L. V., Pizetta, L. C., \& Silva, D. A. (2017). Concordância entre parâmetros antropométricos de obesidade na avaliação do risco cardiovascular em uma amostra de funcionários do setor de alimentação coletiva. Revista Brasileira de Pesquisa em Saúde/Brazilian Journal of Health Research, 19(3), 128-134. https://periodicos.ufes.br/rbps/article/view/19575/13135

Gakidou, E., Afshin, A., Abajobir, A. A., Abate, K. H., Abbafati, C., Abbas, K. M., ... \& Duncan, S. (2017). Global, regional, and national comparative risk assessment of 84 behavioural, environmental and occupational, and metabolic risks or clusters of risks, 1990-2016: a systematic analysis for the Global Burden of Disease Study 2016. The Lancet, 390(10100), 1345-1422. https://doi.org/10.1016/S0140-6736(17)32366-8

Gordon, C. C., Chumlea, W. C., \& Roche, A. F. (1988). Stature, recumbent length, and weight. Anthropometric standardization reference manual. Human kinetics Books.

Harsha, D. W., \& Bray, G. A. (2008). Response to Weight Loss and Blood Pressure Control: The Pro Side. Hypertension,51(6), 1420-1425. https://doi.org/10.1161/HYPERTENSIONAHA.107.094011

Jayedi, A., Rashidy-Pour, A., Khorshidi, M., \& Shab-Bidar, S. (2018). Body mass index, abdominal adiposity, weight gain and risk of developing hypertension: a systematic review and dose-response meta-analysis of more than 2.3 million participants. Obesity reviews, 19(5), 654-667. https://doi.org/10.1111/obr.12656

Koliaki, C., Liatis, S., \& Kokkinos, A. (2019). Obesity and cardiovascular disease: revisiting an old relationship. Metabolism, 92, 98-107. https://doi.org/10.1016/j.metabol.2018.10.011

Loureiro, N. S. D. L., Amaral, T. L. M., Amaral, C. D. A., Monteiro, G. T. R., Vasconcellos, M. T. L. D., \& Bortolini, M. J. S. (2020). Relação de indicadores antropométricos com fatores de risco para doença cardiovascular em adultos e idosos de Rio Branco, Acre. Revista de Saúde Pública, 54, 24. https://doi.org/10.11606/s1518-8787.2020054001088

Malachias, M. V. B., Ferreira Filho, S., Souza, W. K. S. B., Ribeiro, J. M., Miranda, R. D., \& Jardim, T. S. V. (2016). 7th Brazilian Guideline of Arterial Hypertension: Chapter 11-Arterial Hypertension in the elderly. Arquivos brasileiros de cardiologia, 107(3), 64-66. http://dx.doi.org/10.5935/abc.20160161

Malta, D. C., Bernal, R. T. I., Andrade, S. S. C. D. A., Silva, M. M. A. D., \& Velasquez-Melendez, G. (2017). Prevalência e fatores associados com hipertensão arterial autorreferida em adultos brasileiros. Revista de Saúde Pública, 51, 11s. https://doi.org/10.1590/S1518-8787.2017051000006

Nascimento, R. L., Carneiro, M. V. O., de Moraes, J. F. V. N., Moreira, S. R., Souza, A. S., Gomes, M. F. S., ... \& da Silva, L. (2017). Relação entre indicadores antropométricos e pressão arterial em homens e mulheres sedentárias. Revista Brasileira de Pesquisa em Ciências da Saúde, 3(2), 21-27. http://dx.doi.org/10.6084/m9.figshare.8198822

Nascimento, R. L., Carvalho, F. O., Saraiva, L. C., dos Santos Batista, K. G., de Moraes Silva, G., \& de Souza Araujo, F. (2021). Associação de indicadores antropométricos com variáveis hemodinâmicas, glicemia e aptidão física de adultos. Saúde e Pesquisa, 14(1), e8113-e8113. https://doi.org/10.17765/21769206.2021v14n1.e8113

Oliveira, G. M. M. D., Mendes, M., Malachias, M. V. B., Morais, J., Moreira Filho, O., Coelho, A. S., ... \& Fernandes, M. (2017). 2017 Guidelines for Arterial Hypertension Management in Primary Health Care in Portuguese Language Countries. Arquivos brasileiros de cardiologia, $109(5)$, 389-396. https://doi.org/10.5935/abc.20170165

Pitanga, F. J. G. (2011). Antropometria na avaliação da obesidade abdominal e risco coronariano. Revista Brasileira de Cineantropometria \& Desempenho Humano, 13(3), 238-241. https://doi.org/10.5007/1980-0037.2011v13n3p238

Pohl, H. H., Arnold, E. F., Dummel, K. L., Cerentini, T. M., Reuter, É. M., \& Reckziegel, M. B. (2018). Indicadores antropométricos e fatores de risco cardiovascular em trabalhadores rurais. Revista Brasileira de Medicina do Esporte, 24(1), 64-68. https://doi.org/10.1590/1517-869220182401158030

Santos Dias, G., Costa, M. C. B., das Neves Ferreira, T., dos Santos Fernandes, V., da Silva, L. L., Júnior, L. M. S., ... \& Heliotério, M. C. (2021). Fatores de risco associados à Hipertensão Arterial entre adultos no Brasil: uma revisão integrativa. Brazilian Journal of Development, 7(1), 962-977. https://doi.org/10.34117/bjdv7n1-064

Santos, G. C., Campos, W., Faria, W. F., Silva, J. M., Bozza, R., Mascarenhas, L. P. G., ... \& Neto, A. S. (2020). O tempo sentado está associado aos fatores de risco cardiometabólicos em adolescentes? Revista Brasileira de Atividade Física \& Saúde, 25, 1-7. https://doi.org/10.12820/rbafs.25e0132

Saraiva, L. C., de Brito Gomes, J. L., de Araujo, I. S., dos Santos, G. A., Siqueira, A. A., de Moraes, J. F. V. N., \& Carvalho, F. O. (2020). Análise cardiovascular e antropométrica de intervenção com apoio tecnológico em hipertensos: ensaio clínico randomizado. Saúde e Pesquisa, 13(4), 851-859. https://doi.org/10.17765/2176-9206.2020v13n4p851-859

Silva, D. A. S., Pelegrini, A., Pires-Neto, C. S., Vieira, M. F. S., \& Petroski, E. L. (2011). O antropometrista na busca de dados mais confiáveis. Revista Brasileira de Cineantropometria \& Desempenho Humano, 13(1), 82-85. https://doi.org/10.5007/1980-0037.2011v13n1p82

Slaughter, M. H., Lohman, T. G., Boileau, R. A., Stillman, R. J., Van Loan, M., Horswill, C. A., \& Wilmore, J. H. (1984). Influence of maturation on relationship of skinfolds to body density: a cross-sectional study. Human biology, 681-689. https://www.jstor.org/stable/41463613

Topouchian, J. A., El Assaad, M. A., Orobinskaia, L. V., El Feghali, R. N., \& Asmar, R. G. (2005). Validation of two devices for self-measurement of brachial blood pressure according to the International Protocol of the European Society of Hypertension: the SEINEX SE-9400 and the Microlife BP 3AC1-1. Blood pressure monitoring, 10(6), 325-331. 
Research, Society and Development, v. 10, n. 7, e25310716603, 2021

(CC BY 4.0) | ISSN 2525-3409 | DOI: http://dx.doi.org/10.33448/rsd-v10i7.16603

World Health Organization. World Obesity Day. 2020. https://www.who.int/news-room/events/detail/2020/03/04/default-calendar/world-obesity-day

Vrettos, I., Voukelatou, P., Pappa, E., Beletsioti, C., Papadopoulos, A., \& Niakas, D. (2020). Increased body mass index and hypertension: An unbreakable bond. International Journal of Preventive Medicine, 11. 10.4103/ijpvm.IJPVM_218_18

Whelton, P. K., Carey, R. M., Aronow, W. S., Casey, D. E., Collins, K. J., Dennison Himmelfarb, C., ... \& Wright, J. T. (2018). 2017 ACC/AHA/AAPA/ABC/ACPM/AGS/APhA/ASH/ASPC/NMA/PCNA guideline for the prevention, detection, evaluation, and management of high blood pressure in adults: a report of the American College of Cardiology/American Heart Association Task Force on Clinical Practice Guidelines. Journal of the American College of Cardiology, 71(19), e127-e248. https://doi.org/10.1016/j.jacc.2017.11.006 